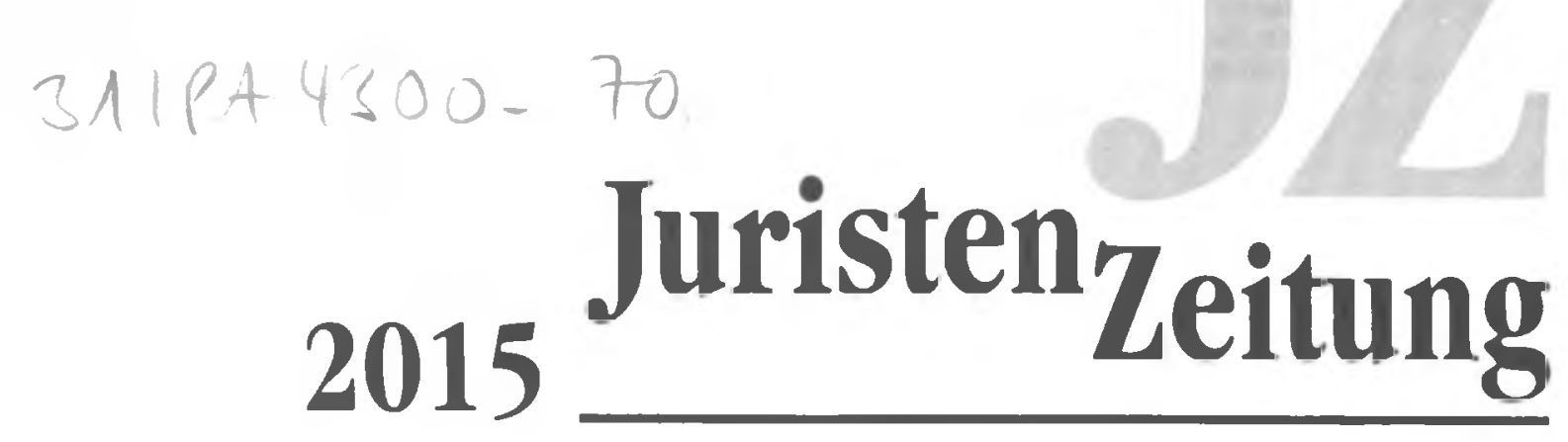

Redaktion

Martin Idler.

72074 Tübingen, Wilhelmstraße 18

Telefon (0 7071) 923-0 oder 923-52, Telefax (0 70 71) 923-67

e-mail: jz@mohr.de

\title{
Herausgeber
}

Professor Dr. Dr. Eric Hilgendorf, Würzburg

Professor Dr. Matthias Jestaedt, Freiburg i.Br.

Professor Dr. Herbert Roth, Regensburg

Professor Dr. Astrid Stadler, Konstanz

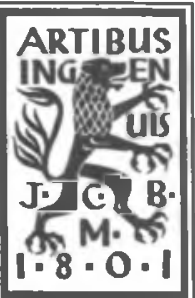

Mohr Siebeck 
Professor Dr. Michael Kubiciel, Köln*

\section{Einheitliches europäisches Strafrecht und vergleichende Darstellung seiner Grundlagen}

\begin{abstract}
Franz von Liszt stand im Zentrum einer vor einhundert Jahren geführten Debatte um die Schaffung eines mitteleuropäischen Strafrechts. Das in Vergessenheit geratene Kapitel europäischer Rechtsgeschichte hat die Ziele. Mittel und Umsetzungsstrategien der heutigen Strafrechtsangleichung in Europa teils bis ins Detail ausbuchstabiert. Darüber hinaus zeigt es den Nutzen eines historischen Grundlagenvergleichs, der gemeinsame Strafrechtsüberlieferungen sichtbar machen und das Europäische Strafrecht an paneuropäische Traditionsbestände zurückbinden kann. Soll das supranationale Strafrecht auf eine - nicht nur in kompetenzieller Hinsicht - solide Grundlage gestellt werden, ist Liszts "Strafgesetzgebung der Gegenwart in vergleichender Darstellung "fortzuschreiben.
\end{abstract}

\section{Kriegseuphorie und Friedensvorsorge}

Im Herbst 1914 gaben sich viele, die "wachsam, aber blind“ in den Krieg getaumelt waren, ${ }^{1}$ noch dem Gedanken an einen glorreichen Kampf und segensreichen Sieg hin. Die Erleichterung, ja Freude über den Kriegsausbruch bei Politikern und Generälen ist häufig beschrieben worden; ${ }^{2}$ Gleiches gilt für die Haltung von Intellektuellen, die wie Thomas Mann ihren "Gedankendienst mit der Waffe ${ }^{\alpha}$ leisteten, ${ }^{3}$ oder Dichtern, die "schockweise [...] Krieg auf Sieg reimten", wie Stefan Zweig entsetzt konstatierte. ${ }^{4}$ Selten beschrieben und kaum erforscht ist hingegen die Haltung der deutschen Rechtswissenschaft zum 1. Weltkrieg, ${ }^{5}$ obgleich Rechtswissenschaftler im Kaiserreich über beträchtliches Ansehen und soziales Kapital verfügten, das heißt eine gesellschaftlich bedeutsame Gruppe bildeten. ${ }^{6} \mathrm{Zu}$ den einflussreichsten unter den "german mandarins" gehörte Franz von Liszt. Der Kriminalist, Völkerrechtler und Reichstagsabgeordnete hatte im September 1914 das berühmt-berüchtigte "Manifest der 93“ unterzeichnet, das die Schuld Deutschlands am Kriegsausbruch sowie deutsche Kriegsverbrechen in Belgien abstreitet

\footnotetext{
- Der Autor ist geschäftsführender Direktor des Instituts für Strafrecht und Strafprozessrecht der Universität zu Köln und Inhaber des Lehrstuhls für Strafrecht, Strafrechtstheorie und Strafrechtsvergleichung. - Der Text basiert auf cinem Vortrag, den der Verf. auf einer Franz von Liszt gewidmeten Tagung an der Universität Augsburg im September 2014 gehalten hat. Die Beiträge werden in einem von Amd Koch und Martin Löbnig herausgegebenen Band erscheinen.

1 Clark, Die Schlafwandler, 2014, S. 718.

2 Zulerzt von Clark (Fn. 1), S. 705 ff., der zugleich eindrucksvoll zeigt, dass die Kriegsbegeisterung bei jenen, die es anging (den Soldaten und ihren Angehörigen), weit weniger groß war, als dies in früheren Schilderungen des August 1914 den Anschein hatte.

3 Mann, Betrachtungen eines Unpolitischen, 2004, Vorrede, S. 31, 41

4 Zweig, Die Welt von gestern, 2000, S. 246, 263. Allgemein zur Haltung der Intellektuellen Mommsen MGZ 59 (2000), 125, $126 \mathrm{f}$.

5 Auf diese Forschungslücke weist hin Kesper-Biermann, in; dies./Overath (Hrsg.), Die Internationalisierung in der Geschichte von Strafrechtswissenschaft und Kriminalpolitik, 2005, S. 85, 98 (mit Anm. 38). Siehe aber Hermann, Das Standardwerk. Franz von Liszt und das Völkerrecht, 2001 S. $102 \mathrm{ff}$., zu Liszt und anderen Völkerrechtlern; von Mayenburg, Kriminologie und Strafrecht vom Kaiserreich bis zum Nationalsozialismus, 2006, S. 243 ff.; Ringer, Die Gelehrten, 1987.

6 V l. dazu Ringer (Fn. 5), S. 12 ff., 33 ff.; Sobn Forschung \& Lehre 2014 $736 \mathrm{ff}$
}

und zur Verteidigung der deutschen Kultur aufruft. Das Geleitwort seiner ebenfalls im Herbst 1914 publizierten Monographie "Ein mitteleuropäischer Staatenverband als nächstes Ziel der deutschen auswärtigen Politik“ schlägt euphorische Töne an und stilisiert den Krieg zu einem "Gesundbrunnen des Volkes".7 Vorbei sei die trübe Zeit politischen Niederganges, man verspüre in diesen Tagen „eine Lust zu leben“. Doch wolle man daheim nicht müßig sitzen, während die Söhne draußen kämpften, sondern mit der Feder für die Größe des Reiches sorgen: „Denn auch sie ist ein Schwert, das wir führen zu unseres Volkes Wohlfahrt."

Des Volkes künftige Wohlfahrt hängt nach Liszts fester Überzeugung von der Sicherung des Friedens nach dem Sieg der Mittelmächte ab. ${ }^{8}$ Liszt, einst Anhänger Bismarcks, ${ }^{9}$ sieht in der Schaffung einer Gemeinschaft mitteleuropäischer Staaten den besten Weg zu einem dauerhaften Frieden in Europa. Diese Gemeinschaft müsse ein Gegengewicht gegen die Großmächte bilden. Die Parole laute: ${ }_{n}$ Schutz gegen England ${ }^{\prime}{ }^{10}$ Der Kern eines mitteleuropäischen Staatenverbandes war klar konturiert; ${ }^{11}$ ihn sollten, nicht überraschend, Deutschland und Österreich-Ungarn bilden. ${ }^{12}$ Unter günstigen Verhältnissen, so Liszt, werde dieses Kerneuropa sich zu einem großen kontinentaleuropäischen Staatenverband auswachsen, ${ }^{13}$ dem die Niederlande, die skandinavischen Länder, Italien, die Schweiz und die "europäische Türkei" angehören könnten. ${ }^{14}$ Der Gedanke eines politisch, wirtschaftlich und rechtlich zusammenrückenden Mitteleuropas machte - als Schlagwort - während des 1. Welrkriegs zwar Karriere. ${ }^{15} \mathrm{Zu}$ konkreten Ergebnissen führte er bis zum Kriegsende indes nicht. ${ }^{16}$ Mit seiner Idee einer "Gesellschaftsbildung" 17 der mitteleuropäischen Nationen zum Zweck der Friedenssicherung hatte Liszt jedoch jenen Gedanken formuliert, der ein halbes Jahrhundert später hinter der Gründung des Europarates und der Europäischen Gemeinschaften stand. ${ }^{18}$ Diese sollten Westeuropa einen und als Gegengewicht zum sowjetischen Machtblock fungieren.

7 Dazu und zum Folgenden ข. Liszt, Ein mitteleuropäischer Staatenverband als nächstes Ziel der deutschen auswärtigen Politik, 1914, Geleitwort: Zwischen Krieg und Frieden, S. If. (gemeinsam unterzeichnet mit Georg Irmer und Karl Lamprecht).

8 ข. Liszt (Fn. 7), S. 5. Eine sonnige Zukunft" sieht auch $v$. Hentig ZStW 29 (1914), 499.

9 Radbruch, Elegantiae Iuris Criminalis, 1950, S. 208, $211 \mathrm{f}$.

10 v. Liszt (Fn. 7), S. 21. Im Folgenden verweist er zudem auf den amerikanischen Statenverband als globalen Konkurrenten (a.a.O., S. 38 f.) Siehe zum Ganzen Herrmann (Fn. 5), S. 125 f.

11 Von einem in seinen geographischen Bezügen diffusen Mitteleuropagedanken spricht hingegen v. Mayenburg, in: Dust/Linder et al. (Hrsg.), Rechtstransfer in der Geschichte, 2007, S. 143, 144

12 ข. Liszt (Fn. 7), S. 27.

13 Ebd., S. 18.

14 Ebd., S. $30 \mathrm{ff}$.

15 Stellvertretend für die zeitgenössische Literatur Liebmann DJZ 1916 , 208 ff.; Naumann, Mitteleuropa, 1915. Zusammenfassend Festl-Wietek, Die deutsch-österreichische Rechtsangleichung, 1989, S. $52 \mathrm{ff}$.

16 Festl-Wietek (Fn. 15), S. 56 f., $70 \mathrm{ff}$.

17 v. Liszt (Fn. 7), S. 27

18 Zur Gründung dieser Institutionen Hattenbawer, Europäische Rechtsgeschichte, 4. Aufl. 2004, S. 869 ff. 
Selbst Liszts Überlegungen zur institutionellen Ausgestaltung des mitteleuropäischen Staatenverbundes finden sich in der Konfiguration der Europäischen Union wieder. So schlägt er vor, dass Verträge sowohl den „Besitzstand ${ }^{“}$ der Staaten wahren als auch die „Einheitlichkeit des wirtschaftlichen Lebens “ gewährleisteten, das heißt einen gemeinsamen Markr schaffen solien. ${ }^{19}$ Zudem sah er eine ständige und eine regelmäßig zusammentretende Konferenz vor, um gemeinsame Angelegenheiten zu beraten - Funktionen, die heute die Europäische Kommission sowie der Rat der Europäischen Union als kontinuierlich arbeitende Organe bzw. der punktuell zusammentretende Europäische Rat der Staatsund Regierungschefs ausüben.

\section{Kulturgemeinschaft und Strafrechtsvereinheitlichung}

Liszt, der seine Kindheit und Jugend in Österreich-Ungarn mit seinen Nationalitätenproblemen verlebt hatte, ${ }^{20}$ war sich bewusst, dass Verträge und Delegiertenkonferenzen die innere Stabilität eines Staatenverbundes nicht garantieren können. Wie aber können jene Zentrifugalkräfte ausgeglichen werden, die in jedem Staatenverbund entstehen? Diese Frage stellte sich Österreich-Ungarn nicht anders als heute der Europäischen Union. Auch Liszt musste sie beantworten, sollte sein mitteleuropäischer Staatenverbund die ihm zugedachte Friedenssicherungsfunktion erfüllen. Liszts Lösung dieses institutionellen Kardinalproblems findet sich in einem weithin vergessenen Text. Er erschien Anfang 1917 in der von ihm herausgegebenen "Zeitschrift für das gesamte Strafrecht". Unter der Überschrift „Einheitliches mitteleuropäisches Strafrecht" verlangt Liszt eine "einheitliche Strafgesetzgebung für die Mittelmächte als eine Aufgabe, nicht der unmittelbaren Gegenwart, wohl aber der allernächsten $\mathrm{Zu}$ kunft." 21 Diese Vereinheitlichung solle der "zielbewußten Stärkung und Weiterbildung der heute bereits vorhandenen Kulturgemeinschaft ${ }^{*}$ der Mittelmächte dienen und "für alle Zukunft die Festigung und Vertiefung des politischen Bündnisses gewährleiste(n). ${ }^{a} 22$

Liszts Text gehört zu einer Serie von Veröffentlichungen, die eine Angleichung des Rechts, namentlich des Handelsund Wirtschaftsrechts, in Deutschland und Österreich-Ungarn propagiert. ${ }^{23} \mathrm{Zu}$ einem nennenswerten sachlichen Ertrag führte aber lediglich die Debatte um die Vereinheitlichung des Strafrechts. ${ }^{24}$ Denn Liszts Aufsatz steht im Zentrum weiterer Arbeiten zur Strafrechtsvereinheirlichung. Ihm vorausgegangen waren fünf Aufsätze, ${ }^{25}$ auf Liszt antworteten unter anderem vier längere Abhandlungen in der "Zeitschrift für das gesamte Strafrecht" ${ }^{\circ}{ }^{26}$ Allesamt eilten sie ihrer Zeit weit voraus. Erst Anfang der 1960er-Jahre crwachte im Europarat wieder die Initiative, ein in den Grundzügen $^{*}$ einheitliches europäisches Strafrecht zu schaffen. ${ }^{27}$

19 Dazu und zum Folgenden v. Liszt (Fn. 7), S. 35, 37.

20 Radbruch (Fn. 8), S. 209. Zum Verhältnis von Lisze zu Österreich umfassend Moll ZStW 81 (1969), 660ff.

21 v. Liszt ZStW 38 (1917), 1, 4.

22 ข. Liszt ZStW 38 (1917), 1, 2.

23 Siche etwa Schiffer DJZ 1916, $457 \mathrm{ff}$; Klein DJZ 1916, 649ff. Weitere Nachweise bei v. Mayenburg (Fn. 11), S. $143 \mathrm{f}$

24 So Festl-Wietek (Fn. 15), S. 73.

25 Gleispach DStrZ 1916, $107 \mathrm{ff}$.; Vámbéry DStrZ 1916, $195 \mathrm{ff}$; Kabl DStrZ 1916, $275 \mathrm{ff}$.; ders. JW 1917, 317 ff.; Hoegel DJZ 1916, $1020 \mathrm{ff}$. 26 Finger ZStW 38 (1917), 319ff.; Goldschmide ZStW 38 (1917), $417 \mathrm{ff}$.; Mittermaier ZStW 38 (1917), $347 \mathrm{ff}$.; Delaquis ZStW 39 (1918), $276 \mathrm{ff}$

27 Dazu Grützner NJW 1961, 2185, 2186, der den Europarat bereits nauf dem Wege zu einem Europäischen Strafrecht" wähnt (a. a. O., S. 2190). - Zu
Sie blieb ohne Erfolg. Nennenswerte Aktivitäten zur Strafrechtsharmonisierung entfaltete erst die EU ab Mitte der 1990 er Jahre. ${ }^{28}$

Obgleich die Rechtsgeschichte seit einigen Jahren verstärkt nach Anknüpfungspunkten für eine "Traditionsbegründung ${ }^{\text {“ }} 29$ des europäischen Rechts befragt wird, ${ }^{30}$ findet die von Liszt und anderen geführte Debatte um das mitteleuropäische Strafrecht nur in vereinzelten (rechtshistorischen) Arbeiten kurze Erwähnung. ${ }^{31}$ Im Folgenden wird dieser erste Anlauf zu einer Harmonisierung des Strafrechts in Europa ausführlicher vorgestellt und kontextualisiert. Dabei werden ideengeschichtliche Entwicklungslinien deutlich, die bis in die Gegenwart reichen (III.). Diese Linien nachzuzeichnen heißt jedoch nicht, einen „Rechtfertigungsnarrativ" für die Europäisierung des Strafrechts schreiben zu wollen. Vielmehr macht gerade Liszts Text straf- und freiheitstheoretisch problematische Implikationen einer Strafrechtsharmonisierung deutlich (IV.). Indes ist in Liszts (Euvre auch ein methodischer Weg vorgezeichnet, um derartige Gefahren zu bannen: der Rechtsvergleich. ${ }^{32}$ Ein rechtshistorisch fundierter Vergleich strafrechtstheoretischer Grundlagen könnte jene materiellen Grenzen einer Strafrechtsvereinheitlichung deutlich machen, die sich an den Kompetenzvorschriften des AEUV und der Grundrechte-Charta gerade nicht ablesen lassen (V.). ${ }^{33}$

\section{Das Projekt einer mitteleuropäischen Strafrechtsvereinheitlichung}

Im 19. Jahrhundert wurde zwar verstärkt grenzüberschreitend und rechtsvergleichend nach Antworten auf kriminalpolitische Fragen gesucht, gleichwohl galt die Strafgesetzgebung als "domaine réservé ${ }^{\alpha}$ des Nationalstaates. ${ }^{34}$ Noch im Jahr 1889 betonte Liszt, dass jede Strafgesetzgebung "nationalen Charakter " tragen und sich in die Eigenart jenes Staates einfügen müsse, für dessen Volk sie bestimmt sei. ${ }^{35}$

kurzzeitigen Bestrebungen im Österreich der 1920er, die österreichische Strafrechisreform an die deutsche zu koppeln, sowie zur Diskussion um eine punktuelle Strafrechtsharmonisierung innerhalb der "Association internationale de droit pénal" ${ }^{*}$, s. Festl-Wietek (Fn. 15), S. 164 ff.; v. Mayenburg (Fn. 11), S. $147 \mathrm{ff}$.

28 Stalt vieler Esser, Europäisches Strafrecht, 2014, \$1 Rn. 10

29 So für das Privatrechi Jansen JZ 2006, 536.

30 Umfassend Schorkop JZ 2014, $421 \mathrm{ff}$.

31 Festl-Wietek (Fn. 16), S. 73-75; ข. Mayenburg (Fn. 11), S. 143-146. Punktuelle Bezugnahmen bei Bacigalwpo ZStW 116 (2004), 326, 328; Kubiciel GA 2010, 99, 100; Mansdörfer HRRS 2010, 11, 16 (dort Anm. 49).

32 Siehe vor allem $v$. Liszt ZStW 26 (1906), $552 \mathrm{ff}$, dessen Annahme, an der geschichtlichen Entwicklung lasse sich das richrige künfrige, weil zweckmäßige Recht, ablesen (a. a.O., S. 555 f.), hier jedoch nicht geteilt wird (siehe unten IV.).

33 Die Bedeutung einer Grundlagenvergleichung für die Strafrechtsharmonisierung betonen Frisch, in: Festschrift für Greve, 2008, S. 139, 156; Kubiciel ZIS 2010, 742, 744 ff.; ders. Rechiswissenschaft 2012, 212, 217 ff.; Weißer GA 2014, 433, 452. Zur Bedeutung der Rechtsvergleichung für die Europäisierung des Strafrechts statt vieler Beck/Burchard/Fateh-Moghadam, in: dies. (Hrsg.), Strafrechtsvergleichung als Problem und Lösung, 2011, S. 5; Hauck, ebd., S. 255 ff. Ferner Ambos Maastriche Journal of European and Comparative Law 12 (2005), 173 ff.; Eser, in: Festschrift für Kaiser, 1998, S. 1499 ff.; Sieber Rechtstheorie 41 (2010), 151, $181 \mathrm{ff}$.; ders. ZStW 119 (2007), 1, 10ff.; Vogel JZ 2012, 25, 29 f.; Weißer, Tàterschaft in Europa, 2011, S. 10.

34 Siehe dazu Böse, in: ders. (Hrsg.) Enzyklopādie Europarecht, Bd. 9: Europäisches Strafrecht, 2013, \1 Rn.4; Jescheck ZStW 98 (1986), 1, 3 f, Kesper-Biermann/Overath, in: dies. (Fn. 5), S. 10; v. Mayenburg (Fn. 11), S. $139 \mathrm{ff.;}$ Sieber, in: ders./Salzger/v. Heintschel-Heinegg (Hrsg.), Europäisches Strafrecht, 2. Aufl. 2014, Einführung Rn. $104 \mathrm{ff}$.; Wetzell, Criminal Law Reform in Imperial Germany, 1991, S. 43 ff.

35 v. Liszt ZStW 9 (1889), 363, 367. 
Fünf Jahre später, in seiner Einführung zu der rechtsvergleichenden Studie "Das Strafrecht der Staaten Europas" deutet er jedoch als ,weiteres Ziel [...] die Ausarbeitung eines europäischen Strafgesetzbuchs ${ }^{\star}$ an, das ${ }_{n}$ gar nicht so utopisch" sei, wie es auf den ersten Blick erscheine. ${ }^{36}$ Vor dem "international gleichen Charakter unseres Verbrechertums" käme den ehrwürdigen Überlieferungen der Strafgesetzbücher nur ein geringes Gewicht zu. ${ }^{37}$ Gehör fanden diese Bemerkungen zunächst nicht. Zwei Jahrzehnte später, mit dem Ausbruch des 1 . Weltkrieges, sollte sich das ändern: Aus der Utopie eines einheitlichen europäischen Strafrechts wurde der Gegenstand einer ernstzunehmenden kriminalpolitischen Debatte.

\section{1. „Auf zur Vereinheitlichung des Strafrechts!”}

Der Anstoß ging nicht von dem damals bereits 65jährigen Liszt aus, sondern von dem deutschnational (und später nationalsozialistisch) gesinnten Graf Gleispach. Der Wiener Rechtslehrer nennt die Friedenssicherung nach dem Krieg als Ziel einer (Straf-)Rechtsangleichung und betont, dass diese eine "Vereinigung aller Kräfte“, ja "möglichste Einheitlichkeit unter den Bundesgenossen “ voraussetze. ${ }^{38}$ Insbesondere gleiches Recht fördere "die Gefühle des Zusammenhalts" und könne diese sogar selbst erzeugen, wenn die vom Recht geregelten tatsächlichen Verhältnisse nicht zu verschieden seien. ${ }^{39}$ Als weitere Ziele (wenn man will: Ziele zweiter Ordnung) benennt Gleispach die Erleichterung des Rechtshilfeverkehrs ${ }^{40}$ und des grenzüberschreitenden $W_{a}$ renaustausches, gehörten Strafvorschriften doch zu den "bejahenden und verneinenden Produktionsbedingungen ".+1 Schließlich erhofft sich Gleispach auch einen wissenschaftlichen Mehrwert, weil durch eine Rechtsangleichung "die ganze geistige Arbeit, die ein Land leistet, unmittelbar auch dem anderen zu gute käme ${ }^{\alpha} .{ }^{42} \mathrm{Da}$ der Zeitpunkt, „um dem idealen Ziel der Rechtsgleichheit näher zu kommen," so günstig sei wie nie, müsse nun ein ernsthafter Versuch zur Angleichung des Strafrechts gewagt werden. ${ }^{43}$ Hinsichtlich großer Teile des Allgemeinen Teils sowie der wirtschaftlich und praktisch wichtigsten Tatbestände ließe sich in „einer gewissen, nicht allzu langen Zeit ${ }^{*}$ Übereinstimmung erzielen." Daher gelte: "Auf zur Vereinheitlichung des Strafrechts! ${ }^{45}$

\section{2. .Wasser im Wein der Begeisterung"}

Dic Reaktionen waren verhalten - und zwar zu Recht. Denn Gleispach führte für dic von ihm behaupteten praktischen Vorteile der Rechtsvereinheitlichung keine Belege an. Insbesondere sein Argument, eine Rechtsharmonisierung generiere einen wissenschaftlichen Mehrwert, überzeugt nicht. Ganz abgesehen davon, dass auch und gerade der Vergleich verschiedener Rechtssysteme wissenschaftlich inspirierend

\footnotetext{
36 v. Liszt, in: Internationale Kriminalistische Vereinigung (Hrsg-), Das Strafrecht der Staaten Europas, 1894, S. XII.

37 Ebd., S. XXI

38 Gleispach DStrZ 1916, 107, 108.

39 Gleispach DStrZ 1916, 107, 110.

40 Diesbezūglich skeptisch Mitcermaier ZStW 38 (1917), 347, 350.

41 Gleispach DStrZ 1916, 107, 109. Ähnlich Hoegel DJZ 1916, 1020, 1022. 42 Gleispach DStrZ 1916, 107, 108f.; Mittermaier ZStW 38 (1917), 347, $350 \mathrm{f}$.

43 Gleispach DStrZ 1916, 107, 111

44 Gleispach DStrZ 1916, 107,113 ff. (Zitat auf S. 117).

45 Gleispach DStrZ 1916, 107,117.
}

sein kann, ${ }^{46}$ impliziert Gleispach, das Recht sei zu vereinheitlichen, um sich gemeinsam mit ihm beschäftigen zu können - ein zumindest gewagter Schluss. ${ }^{47}$ Vor allem aber waren Gleispach die rechtspolitischen Fallstricke, die jede umfassende Änderung des Strafrechts mit sich bringt, kaum ein Wort wert, obgleich diese schon die Arbeit an der deutschen Strafrechtsreform behindert hatten. Eine Antwort auf die naheliegende Frage, wie diese Fallstricke durch eine Reform gleich mehrerer Strafrechtsordnungen übersprungen werden könnten, blieb Gleispach schuldig.

Der Budapester Universitätsdozent Vámbéry lenkt in seiner Antwort denn auch den Blick auf die Schwierigkeiten eines solch ambitionierten Vorhabens. Wenn der Wunsch nach einer Vereinheitlichung des Strafrechts mehr sein solle als eine "Kundgebung waffenbrüderlicher Gefühle ", müssten auf Grund "sachlicher Erwägungen" die Grenzen bestimmt werden, in denen sich eine Harmonisierung überhaupt verwirklichen lasse. ${ }^{48}$ Die wichtigste Grenze bilden nach seiner Überzeugung, die in jedem Land wirkenden „rechtserzeugenden Kräfte". Zu diesen Kräften rechnet Vämbéry offenkundig auch die gesellschaftlichen Wertüberzeugungen der Länder. Er betont jedenfalls die Gefahr, dass ein auf zwischenstaatlicher Ebene gesprochenes "Machtwort" zur Rechtsanpassung den Strafgesetzen die "mächtige Stütze der Volkstümlichkeit" nehme. ${ }^{49} \mathrm{Um}$ dies zu verhindern, sollen die Tatbestände des Besonderen Teils weitgehend unangetastet bleiben; zu unterschiedlich sei das Bedürfnis nach Schutz für Rechtsgüter in den Ländern.50 Lediglich bei Straftatbeständen zum Schutz der militärischen und diplomatischen Sicherheit sowie des Bank-, Börsen, Telegraphen- und Postwesens besteht Vámbéry zufolge die Möglichkeit der Harmonisierung. Hinter dieser Auflistung steckt der Gedanke, dass Angriffe auf diese transnationalen Institutionen deshalb besonders schwer wiegen, weil sich die Folgen nicht auf einen Staat beschränken lassen, sondern alle treffen. Dieser Gedanke findet sich heute in Art. 83 Abs. 1 AEUV, der eine Rechtsangleichung in Bereichen besonders schwerer Kriminalität gestattet, „die aufgrund der Art oder der Auswirkungen der Straftaten oder aufgrund einer besonderen Notwendigkeit, sie auf gemeinsamer Grundlage zu bekämpfen, eine grenzüberschreitende Dimension haben" ${ }^{5} 51 \mathrm{Zu}$ der Strategie, das Strafrecht zum Schutz geteilter Interessen und Werte einzusetzen, passt, dass Vämbéry - eine Idee Liszts aufgreifend - für eine Harmonisierung des Strafanwendungsrechts plädiert. ${ }^{52}$ Aber auch in diesem Zusammenhang mahnt er, sich auf Bereiche zu beschränken, in denen sich die verbündeten Staaten bereits gegenseitigen Schutz ihrer Rechtsgüter gewähren. ${ }^{53}$ Darüber hinaus ließe sich, so Vámbéry, das Strafrecht hinsichtlich jener "verkehrsrechtlichen Regeln" harmonisieren, welche die Staaten bereits weitgehend angeglichen hatten; ${ }^{54}$ diese Primärnormen sollen, so ist Vämbéry zu verstehen, von einheitlichen strafrechtlichen Sanktions-

\footnotetext{
46 Kahl DStrZ 1916, 275, 276.

47 v. Mayenburg (Fn. 11), S. 145: „absurd“

48 Dazu und zum Folgenden Vámbéry DStrZ 1916, 195, 196

49 Vámbéry DStrZ 1916, 195, 196. Relativierend hingegen Hoegel DIZ

1916, 1020, 1022; Goldschmidt ZSrW 38 (1917), 417, 421

50 Vambéry DStrZ 1916, 195, 198.

51 Dazu Salzger, Internationales und europäisches Strafrecht, 6. Aufl. 2013, 59 Rn. 36.

52 Vámbêty DStrZ 1916, 195, 197; v. Liszt, Gutachten für den 16. Deutschen Juristentag 1882, in: ders. (Hrsg.), Strafrechtliche Aufsätze und Vorträge, Bd. 1, 1905, S. 90 ff.

53 Dazu und zum Folgenden Vämberry DStrZ 1916, 195, 197.

54 Vämberry DStrZ 1916, 195, 199.
} 
normen abgesichert werden können. Hier klingt das heute in Art. 83 Abs. 2 AEUV enthaltene Recht zur sogenannten Annexharmonisierung an, der zufolge Strafvorschriften vereinheitlicht werden dürfen, wenn dies zur Durchsetzung einer bereits erfolgten anderen Harmonisierungsmaßnahme unerlässlich ist. ${ }^{55}$ Jenseits dessen sieht Vámbéry nur geringen Spielraum für eine Rechtsangleichung. Er nennt die Regeln auf dem „ethisch farblosen Gebiet der Sicherungsmaßnahmen" 56 sowie Begriffsbestimmungen des Allgemeinen Teils wie den Vorsatz- und Fahrlässigkeitsbegriff. Letzteres solle aber nur zu einer formellen Angleichung von Begriffen führen, soweit diese "mehr Rechtssätze als Lehrinhalte" seien.

Die derart zurückgenommenen Erwartungen an das Projekt einer mitteleuropäischen Strafrechtsangleichung dämpfte der deutsche Rechtswissenschaftler Wilhelm Kabl weiter. In seinem Debattenbeitrag wendet er sich gegen das - von Liszt und Gleispach betonte - Argument, eine "Rechtsverwandtschaft" könne "zuverlässiger Hüter des Burgfriedens unter den Staaten " werden. ${ }^{57}$ Kühl verweist er auf die Erfahrungen der Realpolitiker: Das Wohlverhalten der Staaten hänge von der Übereinstimmung realer Interessen $a b$, nicht von der Einheit des Rechts. Dass es ein allgemeines Bedürfnis nach Bekämpfung von Verbrechen gebe, lässt $K a b l$ als Grund für eine Strafrechtsangleichung nicht gelten: „Denn es kommt darauf an, [...] mit welchen Mitteln geahndet und bekämpft werden soll." ${ }^{58}$ In der Tat können die Ansichten über Art und Umfang des Schutzes auseinandergehen, selbst wenn man sich über die grundsätzliche Schutzwürdigkeit cines Rechts oder einer Institution einig ist. So ist der Wettbewerb in Europa als schutzwürdig anerkannt. Gleichwohl divergieren beispielsweise die Meinungen, ob Straftaten gegen den Wettbewerb, die aus einem Unternehmen heraus begangen werden, auch eine Bestrafung der hinter dem Unternehmen stehenden juristischen Person nach sich ziehen sollen. Divergenzen betreffen jedoch nicht nur dic Art, sondern auch und vor allem den Umfang des Schutzes: Erinnert sei hier nur an die Debarte um die Reichweite eines Verbots der Bestechung im geschäfrlichen Verkehr ( $\$ 299$ StGB). Ferner ist man sich zwar europaweit einig, dass den Gefahren des Terrorismus entschieden begegnet werden soll. Kein Konsens besteht jedoch hinsichtlich der Frage, ob und inwieweit das Strafrecht zu diesem Zweck eingesetzt werden kann. Selbst die von $K a b l$ angedeutete Vereinheitlichung des „Individualrechtsschutzes ${ }^{459}$ hätte erhebliche Hürden zu überwinden, wenn man an die divergierende Ausgestaltung der Tötungsdelikte, des Sterbehilferechts, aber auch der Betrugstatbestände denkt.

\section{Liszts Plädoyer für ein mitteleuropäisches Strafrecht}

Kabl schließt seinen Beitrag mit dem Bedauern, er habe Wasser in den "Wein der Begeisterung für Strafrechtsangleichung" mischen müssen.60 Dics ist mehr als angemessen,

\footnotetext{
55 Statt vieler Kotzur, in: Geiger/Khan/Kotzur, EUV/AUEV, 5. Aufl. 2010, Art. 83 AEUV Rn. 11

56 Vambéry DStRZ 1916, 195, 199.

57 Dazu und zum Folgenden Kabl DStrZ 1916, 275, 277.

$58 K a b l$ DStrZ 1916, 275, 278

59 Kahl DStrZ 1916, 275, 280 ff. Zudem nennt er die Regeln über das Strafanwendungsrecht, den Versuch, die Konkurrenzen, den Strafantrag und die Sicherungsmaßregeln.

60 Kabl DStrZ 1916, 275, 285
}

denn er hat die Idee der Rechtsvereinheitlichung nicht einfach nur relativiert; seine Ausführungen kommen vielmehr einer Ablehnung nahe. ${ }^{61}$ Dem aus Wien erschallenden ${ }_{n}$ Auf zur Vereinheitlichung des Strafrechts“ hielt der Ungar Vámbéry die kulturelle Vorprägung wesentlicher Teile des Strafrechts entgegen. Und die Hoffnung, das Recht könne zu einem „neuen Band“ der Völker werden, dämpfte der Berliner Kabl mit dem Hinweis, gemeinsame Interessen führten Staaten zusammen, nicht Gesetzbücher.

Es bedurfte schon eines Mannes von großem Optimismus und Mut zu kühnen Zielsetzungen, um die Debatte um ein einheitliches europäisches Strafrecht zu beleben. Franz von Liszt war ein solcher Mann. ${ }^{62}$ Auch der Ausbruch des Weltkrieges hatte Liszts Fortschrittsglauben nicht erschüttert, wie die eingangs erwähnte Monographie und Schilderungen aus seiner Umgebung belegen. ${ }^{63}$ Liszt beharrt darauf, dass die Gemeinsamkeit der Kultur und des Rechts die Völker viel stärker und zuverlässiger verbinde als die von Diplomaten geschlossenen Verträge. ${ }^{64} \mathrm{Nach}$ wie vor existierende Unterschiede zwischen den Kulturen sind für Liszt überwindbar (1.). Gerade das Strafrecht ist für ihn ein Mittel zur Nivellierung von Kulturunterschieden (2.).

\section{Historische Argumente}

Dass kulturelle Unterschiede einer erfolgreichen Rechtsrezeption nicht im Wege stehen, ${ }^{65}$ begründet Liszt historisch. Unterschiede der Kultur hätten weder der Rezeption des französischen Strafrechts durch Preußen ${ }^{66}$ noch der Schaffung eines einheitlichen deutschen Strafrechts für so verschiedene Länder wie Baden und Mecklenburg im Wege gestanden. ${ }^{67}$ Die Beispiele sind jedoch wenig überzeugend. Bei der Rezeption französischen Strafrechts hatten die deutschen Länder erhebliche Entscheidungsspielräume, so dass Kodifikationen wie dem Bayerischen Strafgesetzbuch von 181368 und dem Preußischen Strafgesetzbuch von 1851 ein großes $\mathrm{Maß}$ an Eigenständigkeit zukommt. ${ }^{69}$ Das Reichsstrafgesetzbuch wiederum stand zwar zeitlich gesehen am Anfang des nation building. In politisch-kultureller Hinsicht betrachteten aber große Teile der Bevölkerung die deutschen Einzelstaaten schon vor 1871 als Einheit. Diese Bedingungen waren 1917 schon innerhalb des Vielvölkerstaats Österreich-Ungarn nicht gegeben. ${ }^{70}$ Hier hätte die Rechtseinheit zwischen Deutschland und Österreich-Ungarn dem Zusammengehörigkeitsgefühl nicht nachfolgen können, sondern dieses $\mathrm{Ge}$ fühl herbeiführen müssen.
61 Treffend Hoegel DJZ 1916, 1020.

62 Siehe v. Liszt JW 1918, 754, Zu diesen Eigenschaften Hermann NJW 2001, 2854, 2858; v. Hippel ZStW 40 (1919), 529, 531 u. 533; Finger Der Gerichtssaal 87 (1920), 113, 114; Radbruch (Fn. 9), S. 213.

63 So, unter Verweis auf ein Gespräch mir Liszt, Finger Der Gerichtssaal $87(1920), 113,117$.

64 ข. Liszt ZStW 38 (1917), 1, 9f. Insoweit zustimmend Goldschmidt ZStW $38(1917), 417,419$.

65 So auch Mittermaier ZStW 38 (1917), 347, $352 f$

66 v. Liszt, in: Internationale Kriminalistische Vereinigung (Fn.36), S XII.

67 v. Liszt ZStW 38 (1917), 1, 11; zustimmend Goldschmidt ZStW 38 (1917), 417, 421

68 Löhnig, in: Koch/KubicielLöhnig/Pawlik, Feuerbachs Bayerisches Strafgesetzbuch, 2014, S. $81,90 \mathrm{ff}$.

69 Vgl. Mittermaier ZStW 38 (1917), 347, 348

70 Ergänzend weist Hoegel DJZ 1916, 1020 f., darauf hin, dass in Österreich-Ungarn nicht nur zwei, sondern fünf Strafgesetze galten: Neben der österreichischen und ungarischen auch die kroatisch-slawonische, bosnischherzegowinische und die Militärstrafgesetzgebung. 


\section{Strafrechtstheoretische Erwägungen}

Tatsächlich hält Liszt das Strafrecht für besonders geeignet zur Schaffung eines solchen Zusammengehörigkeitsgefühls. Das Recht, so Liszt, sei nicht nur Erzeugnis einer Kultur, sondern auch „einer der einflussreichsten Faktoren für die Gestaltung des Geisteslebens, für die Weiterbildung der Kultur ${ }^{4}{ }^{71}$ Oder prägnant formuliert: Es ist nicht nur „Kulturprodukt“, sondern auch „Kulturhebel“. Die Funkrion als Kulturhebel erfüllt das Strafrecht als $z$ wangsweise sich durchsetzende ,Anleitung zum richtigen Verhalten' und darum auch zum richtigen Werten", es bewirke eine "Erziehung durch angedrohten Zwang". 72 Anders als in seinem Marburger Programm ist die Abschreckung hier kein Mittel, um ${ }_{n}$ die Autorität des übertretenen Gesetzes her(zu)stellen ${ }^{4} .73$ Vielmehr sollen Strafandrohung und -vollstreckung auf die Haltung der Bürger einwirken, anders gewendet: diese ändern. Legt man die Kategorien des Marburger Programms zugrunde, sind die Bürger in einem Staatenverbund mit divergierender Kultur und fehlendem Zusammengehörigkeitsgefühl also „Besserungsbedürftige“. Strafandrohung und Strafzwang sollen sie nicht als Pflichtenmahnung zur äußeren Legalität anhalten, sondern zur Angleichung ihrer inneren, kulturellen Einstellungen. Dies zeigt in voller Schärfe die freiheitsfeindliche Tendenz der Idee, das Strafrecht als Kulturhebel einzusetzen. Zudem überschätzt Liszt die Steuerungskraft des Rechts und unterschätzt, wie stark die Auslegung ein- und derselben Rechtsnorm divergiert, wenn sie in verschiedenartigen Gesellschaften zur Geltung gebracht wird. Die Rechtsvergleichung stellt zum Beweis für diesen Effekt reiches Anschauungsmaterial bereit. Finger berichtet in einer Antwort auf Liszt etwa von "losen Reden", die im vornehmen Oberösterreich als Beleidigung oder Religionsstörung geahndet würden, während einem Richter in Böhmen nicht einmal im Traum einfalle, gegen sie einzuschreiten. ${ }^{74}$

Für Liszt ist das einheitliche mitteleuropäische Strafrecht aber nicht nur ein Instrument zur Durchsuchung homogener Wertvorstellungen in den Gesellschaften der verbündeten Staaten. Liszt sieht es auch als Mittel zur Bekämpfung der Gefahren, die vom grenzüberschreitenden Verkehr zwischen den Staaten ausgehen. Nicht nur Waren überquerten Grenzen, sondern auch „die arbeitsscheuen Schmarotzer am gesellschaftlichen Körper, von den eleganten Dirnen angefangen bis zum bescheidensten Landstreicher ${ }^{\star} .{ }^{75} \mathrm{Zu}$ dem Einwand Kahls, es käme nicht allein auf die Beschreibung der Schutzziele an, Einigkeit müsse auch hinsichtlich des auszuwählenden Mittels - des Strafrechts - bestehen, verliert Liszt kein Wort. Er kann es auch nicht, da nach seinem Verständnis vom Strafrecht als Präventionsrecht zwischen dem Polizei- und dem Strafrecht kein substantieller Unterschied besteht. Sieht man nämlich wie Liszt im Strafrecht ein Instrument des Rechtsgüterschutzes, lassen sich die Besonderheiten des Strafrechts nicht erfassen. ${ }^{76}$ Es ist dann im Wesentichen eine politische Ermessensfrage, zu welchem Mittel der (nationale, zwischenstaatliche oder überstaatliche) Gesetzgeber greift.

71 ข. Liszt ZStW 38 (1917), 1, 3. Mit Differenzierungen, aber im Grundsatz zustimmend, Mittermaier ZStW 38 (1917), 347, $351 \mathrm{f}$.

72 ข. Lisze ZStW 38 (1917), 1, 3 f.

73 Dazu und zum Folgenden v. Liszt ZStW 3 (1883), 1, 43.

74 Vgl. dazu Finger ZSiW 38 (1917), 319, 330 f.

75 v. Liszt ZStW $38(1917), 1,4$

76 Parolik, Das Unrecht des Bürgers, 2012, S. 63; Kubiciel, Die Wissenschaft vom Besonderen Teil des Strafrechts, 2013, S. 159.

\section{Gubernative Strafrechtsvereinheitlichung}

Zur Vorbereitungen einer politischen Entscheidung solle, so Liszt, ohne Zeitverzug eine Kommission von je einem Fachvertreter aus den drei Ländern zusammentreten. Diese Kommission solle einen Gesetzentwurf erarbeiten, der mit den von den Regierungen gewünschten Änderungen in einen völkerrechtlichen Vertrag einfließen könne. Die nationalen Parlamente sollten diesen Vertrag lediglich annehmen oder ablehnen, nicht aber ändern können. ${ }^{77}$ Damit hat Liszt das in Grundzügen - bis heute praktizierte Prinzip der gubernativen europäischen Gesetzgebung vorweg genommen. ${ }^{78}$

\section{Würdigung und Wirkung}

Liszts Auffassung ist eher technokratisch als freiheits- und demokratiefreundlich und weist argumentative Schwächen auf. Gleichwohl waren die Reaktionen auf Liszts Text überwiegend positiv. ${ }^{79}$ Es ginge aber fehl, darin den Ausdruck einer "dezidiert deutsch-nationalen Strafrechtspolitik " zu sehen. ${ }^{80} \mathrm{Zwar}$ schwingen in manchen Arbeiten nationalistische Untertöne mit; bei Gleispach sind sie sogar deutlich vernehmbar. Ungeachtet dessen ist die Debatte im Ganzen ausgesprochen gedankenreich, wie die erstaunlichen Parallelitäten mit dem Europäischen Strafrecht der Gegenwart zeigen.

\section{Vergessen und doch aktuell}

\section{Gründe für die fehlende Rezeption}

Gleichwohl sind die Texte in Vergessenheit geraten. Dies dürfte auf zwei Faktoren zurückzuführen sein. Zunächst brach die Debatte um eine Strafrechtsvereinheitlichung mit Ausbruch des Zweiten Weltkrieges endgültig ab. ${ }^{81}$ Als HansHeinrich Jescheck im Jahr 1953 auf der Strafrechtslehrertagung anlässlich der Gründung der „Montanunion“ und der Vorbereitung einer Europäischen Verteidigungsgemeinschaft zur "Strafgewalt übernationaler Gemeinschaften" referierte, bezog er sich auf die Rheinschifffahrtsakte von 1831 und die nach den Weltkriegen ventilierte Idee einer internationalen Strafgewalt über Kriegs- und Humanitätsverbrechen. ${ }^{82}$ Selbst wenn Jescheck die Texte Listzs und der anderen gekannt hätte, wäre ihm eine Reminiszenz an ein Strafrecht der Mittelmächte in der Stunde der Westbindung gewiss als inopportun erschienen. So geriet die Debatte der Jahre 1916 und 1917 in Vergessenheit. Daran hat sich bis heute nichts geändert. Dies ist auch darauf zurückführen, dass die Forschung zur Geschichte des Europäischen Strafrechts noch an ihrem Anfang steht. ${ }^{83}$ Weil in den letzten Jahrzehnten hauptsächlich um die Kompetenzen der EU auf dem Gebiet des Strafrechts gerungen wurde, ${ }^{84}$ zeichnen viele Darstellungen die Reise "von Rom nach Lissabon" nach. Auf größere, ide-

\footnotetext{
77 ข. Liszt ZStW 38 (1917), 1, 17.

78 v. Bogdandy, Gubernative Rechtsetzung, 1999, S. 4; Grabitz/Hilf/Net tesheim, Das Recht der Europäischen Union, 53. Ergänzungslieferung 2014, Art. 290 AEUV Rn. $16 f$.

79 Goldschmidt ZStW 38 (1917), 417 ff.; Mittermaier ZStW 38 (1917), 347, 348. Ablehnend Finger ZStW 38 (1917), 319, 328 ff.

80 Zu kritisch v. Mayenburg, in: Dust/Linder et al. (Fn. 11), S. $145 \mathrm{f}$

81 Sieber, in: ders.Satzger/v. Heinischel-Heinegg (Fn. 34), Rn.110.

82 Jescheck ZStW 65 (1953), 496, $498 \mathrm{ff}$.

83 Vgl. v. Mayenburg (Fn. 5), S. 137: "stiefmütterliche" Behandlung durch die Rechtshistoriker.

84 Esser (Fn. 28), \$2 Rn. 121 ff.; Satzger (Fn. 51), \$8 Rn. 9.
} 
engeschichtliche Überlieferungszusammenhänge greifen sie hingegen nicht zurück. ${ }^{85}$

\section{Vom Nutzen einer vergleichenden Strafrechtstheorie}

Mit der Konsolidierung der Kompetenzgrundlagen durch den Vertrag von Lissabon ist inzwischen die Kriminalpolitik in das Zentrum der strafrechrlichen Diskussionen gerückt. Verstärkt wird die Rationalisierung der europäischen Kriminalpolitik gefordert und nach Maßstäben für die europäische Strafgesetzgebung gesucht. ${ }^{86}$ Einer verbreiteten rechtspositivistischen Hintergrundüberzeugung entspricht es, diese Maßstäbe in der höchsten Normenordnung zu suchen. So wie in Deutschland das Grundgesetz als Prüfstein der Gesetzgebung dient, wäre dann der Grundrechte-Charta der Maßstab für eine europäische Strafrechtsharmonisierung zu entnehmen. ${ }^{87}$ Aus der Verfassung bzw. der GrundrechteCharta lassen sich zwar subjektive Rechte und objektive Werte ableiten. Nicht ableiten lässt sich aber aus diesen Normenordnungen, wie eine Kollision von Rechten und Werten aufzulösen ist, ${ }^{88} \mathrm{da}$ alle Verfassungswerte grundsätzlichvon gleichem Rang sind. ${ }^{89}$ Die "Verfassung eines modernen, vollpositiven Rechtsstaates" ruht legitimationstheoretisch zwar auf sich selbst, ${ }^{90}$ kann aber nicht aus sich selbst heraus konkretisiert und ausgelegt werden. Wie konkurrierende subjektive Rechte, Werte oder Interessen zu gewichten sind, muss vielmehr mittels einer Abwägung entschieden bzw. anhand des Verhältnismäßigkeitsprinzips geprüft werden. Eine Abwãgung (und damit auch die Prüfung der Verhältnismäßigkeit i.e.S.) ist jedoch ein Verfahren, das auf die Zuführung externer Entscheidungstopoi angewiesen ist. Soll der europäische Gesetzgeber den ihm zugebilligten weiten Ermessensspielraum ${ }^{91}$ nicht nur mit kriminalpolitischen Erwägungen ausfüllen, hat die Rechtswissenschaft strafrechtliche Prinzipien zu benennen, welche bei der europäischen Strafrechtsangleichung zu berücksichtigen sind. Aus dem Primärrecht lassen sich diese - wie gesehen - nicht ableiten, und auch das europäische Sekundärrecht ist für die Suche nach solchen Prinzipien ein schlechter Ort, weist es doch einen fragmentarischen Charakter auf. ${ }^{92}$ Wer eine systematisch-kohärente Kriminalpolitik anstrebt, ${ }^{93}$ muss sich folglich an Strafrechts-

85 Ausnahmen: Böse, in: ders. (Fn. 34), \& / Rn. 2 ff.; Sieber, in: ders./Satzger/o. Heintschel-Heinegg (Fn. 34), Einführung Rn. 13 ff.

86 Dies ist auch das Ziel der European Criminal Policy Initiative ZIS 2010, 697; dazu Salzger ZIS 2009, 691; ders. ZRP 2010, 137. Siehe ferner Kubiciel GA 2010, 99ff.; ders. ZIS 2010, 742, 744 ff.; Meyer EuR 2011, 169, $193 \mathrm{ff}$.; Weigend, in: Festschrift für Zoll, 2012, S. 205, $222 \mathrm{ff}$.

87 So insbesondere Meyer, Strafrechtsgenese in internationalen Organisationen, 2012, S. 774 ff.; Vogel, in: Ambos (Hrsg.), Europäisches Strafrech Post-Lissabon, 2011, S. 41, 54 ff. Vgl. ferner Eser, in: Meyer (Hrsg.), Kommentar zur Charta der Grundrechte der Europäischen Union, 3. Aufl. 2011, Art.49 Rn. 37; T. Walter ZStW 117 (2005), 912, 921 f.; Weigend ZStW 116 $(2004), 275,278$.

88 Näher Röhl/Röhl, Allgemeine Rechıslehre, 2008, S. 64.

89 Ladeur, Kritik der Abwägung in der Grundrechtsdogmatik, 2004,

S. 9f. Zutreffend die europäischen Grundrechte als, teils irrelevant, teils zu unspezifisch" für die Europäisierung des Strafrechts bezeichnend Weigend, in: Zieschang/Hilgendorf/Laubenthal (Hrsg.), Strafrecht und Krimi nalität in Europa, 2002, S. 57, 68.

90 Gärditz, in: Isensee/Kirchbof (Hrsg.), Handbuch des Staatsrechts, Bd. XI, 3. Aufl. 2013, \245 Rn. 40. Umfassend dazu Satzger, Die Europäisierung des Strafrechts, 2001, S. 159 ff.; Weigend ZStW 105 (1993), 774 ff.

91 Meyer (Fn. 87), S. 777 f.; Trstenjak/Beysen EuR 2012, 265, 273. 92 Höpfner/Rüthers AcP 209 (2009), 1, 3.

93 Rat der Europäischen Union, Ratsdokument 11 155/11, S. 2; Mitteilung der Kommission: Auf dem Weg zu einer europäischen Strafrechtspolitik, KOM(2011), 573 endgültig, S. 3 f. Siehe auch Satzger, in: Böse (Fn. 34), $\$ 2$ prinzipien orientieren, die sich nur auf einer vorpositiven Ebene finden lassen. Dort, auf der vorpositiven Ebene, liegt daher die "wissenschaftliche Basis eines übernationalen Strafrechts ${ }^{\alpha 94}$.

Zutritt zu ihr gewährt ein Vergleich strafrechtstheoretischer Grundlagen. Gemeint ist damit ein Rechtsvergleich, der nicht an Normen und ihren Funktionen 95 bzw. den "Kriminaljustizsystemen" 96 haftet, der sich aber auch nicht auf den gesamten vornormativen Bereich der Kultur erstreckt, ${ }^{97}$ sondern einen Ausschnitt der Rechtskultur fokussiert: die Strafrechtstheorie. Bezieht man in diesen Vergleich auch den Prozess der Genese strafrechtlicher Theorien und Prinzipien im 18. und 19. Jahrhundert ein, könnte ein paneuropäischer Traditionsbestand sichtbar werden, ${ }^{98}$ der in einem europäischen Diskurs entstanden, in nationale Strafrechtssysteme eingeflossen und dort in unterschiedlicher Gestalt erhalten geblieben ist. Obgleich prinzipiell-abstrakter Natur, können diese gemeinsamen Strafrechtsüberlieferungen bei der Beantwortung konkreter Fragen wie der nach den Grenzen eines Präventionsstrafrechts, der Bedeutung des Tatprinzips oder den grundsätzlichen Voraussetzungen einer $\mathrm{Zu}$ rechnung fremden Verhaltens helfen und damit das Fundament einer rationalen Kriminalpolitik für den "Raum der Freiheit, der Sicherheit und des Rechts" bilden. Dessen Offenlegung ist umso erforderlicher, je mehr die Strafrechtsangleichung in Europa über punkruelle Assimilierungen einzelner Tatbestände hinausgeht und sich zu einem in der Summe einheitlichen europäischen Strafrecht auswächst. ${ }^{99}$ Das Interesse an der Genese solcher Prinzipien beginnt zwar zu erwachen. ${ }^{100}$ Anders als im Verfassungs-, Verwaltungs- und Zivilrecht steht die Suche nach gemeineuropäischen Strafrechtsprinzipien jedoch noch an ihrem Anfang. 101 Es ist daher an der Zeit, Liszts ${ }_{n}$ Strafgesetzgebung der Staaten Europas" um einen Band mit einem Grundlagenvergleich zu ergänzen und mit einer strafrechtstheoretischen Synthese zu beschließen. Diese Synthese könnte offenlegen, inwieweit die "kulturell voraussetzungsvolle Basisstruktur des Strafrechts ${ }^{\text {" } 102}$ eine einheitlich europäische ist und wie weit die Rechtsangleichung betrieben werden kann.

\section{Lektionen der Debatte um die mitteleuropäisches Strafrechtsvereinheitlichung}

Auch die geschilderte Debatte um ein mitteleuropäisches Strafrecht ist ein Überlieferungszusammenhang, an den sich - positiv wie negativ - anknüpfen lässt. Einerseits werden in den Texten Gegenstandsbereiche skizziert, auf denen auch heute Raum für eine Harmonisierung des Strafrechts ist. So müssen Institutionen wie die staatlichen Verwaltungen sowie das Finanz- und Wirtschaftssystem im politisch und ökonomisch integrierten Raum der EU kohärent geschützt wer-

\footnotetext{
94 Hirsch ZStW 116 (2004), 835, 838.

95 Dazu Fareh-Moghadam, in: Beck/Burchard/Fateh-Moghadam (Fn_33), S. $52 \mathrm{ff}$.

96 logel JZ 2012, 25, 30.

97 Dazu Hilgendorf, in: Beck/Burchard/Falch-Moghadam (Fn. 33), S. 22, 23; Beck, a. 2. O., S. 65, 71.

98 Zum Zusammenhang von Strafrechtsvergleichung und Strafrechtsgeschichte Koch, in: Fesıschrift für Frisch, 2013, S. 1483, 1498.

99 Steigende Anforderungen an die Strafrechtsvergleichung konstatiert

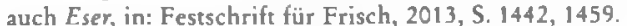

100 Siehe Frisch GA 2007, 251 ff.; Kühl ZStW 109 (1997), 786, $794 \mathrm{f}$.

101 Zur Bedeutung der (geschichtlichen) Rechtsvergleichung für das europäische Verwaltungs- und Privatrecht Heun, in: ders./Schorkopf (Hrsg-), Wendepunkte der Rechtswissenschaft, 2014, S. 9, 26 ff. mit weiteren Nachweisen.

102 Gärditz, in: HStR (Fn. 90), \$245 Rn. 24
} 
den, weil die Folgen ihrer Beschädigungen europaweit spürbar sind. Andererseits ist mit Kabl daran zu erinnern, dass eine grundsätzliche Einigkeit hinsichtlich der Schutzwürdigkeit eines Rechtes oder einer Institution nicht impliziert, dass die Gesellschaften der Mitgliedstaaten derselben Auffassung über Umfang und Art des Schutzes sind. Diesbezügliche Divergenzen sollte der europäische Gesetzgeber nicht ohne Not einebnen, wenn er seinem Strafrecht und seiner Rechtssetzungskompetenz nicht die gesellschaftliche Legitimationsbasis nehmen will, ohne die langfristig nicht auszukommen ist. ${ }^{103}$ Deutlich geworden ist auch eine höchst problematische strafrechtstheoretische Implikation der in vielen Dokumenten der EU zu findenden Erwägung, ein einheitliches Strafrecht könne ein gemeinsames "Rechtsgefühl “ bei Bürgern ${ }^{104}$ oder Normanwendern ${ }^{105}$ erzeugen bzw. zu einer europäischen Identitätsbildung beitragen. ${ }^{106}$ Der Einsatz des Strafrechts als "Kulturhebel ${ }^{*}$ führt nicht nur zu den oben beschriebenen Freiheitsverlusten. Es fragt sich auch, ob er tatsächlich leisten kann, was man sich von ihm verspricht. Zweifel sind angebracht. Der Grund für die Zweifel scheint in einem Gedanken T. S. Eliots auf. Der Dichter, Nobelpreisträger und große Europäer wendete sich kurz nach Ende des 2. Weltkrieges in einer Radioansprache an deutsche Hörer. Eliot stellte - wie Liszt drei Jahrzehnte zuvor - die europäische Schicksalsfrage: Was kann die Völker Europas davon abhalten, sich in einem neuen Krieg aufeinander zu stürzen? Anders als Liszt skizzierte Eliot keine institutionellen Arrangements zwischen den Staaten, sondern erinnerte - ähnlich wie Liszt - an das gemeinsame kulturelle Erbe, insbesondere das christliche. Langfristig werde nur diese Kultur das Zusammenwachsen der Völker fördern und damit den Zusammenhalt der Staaten festigen. Im Unterschied zu Liszt und vielen heutigen Europapolitikern sah Eliot jedoch, dass sich eine gemeinsame europäische Kultur weder mit Hilfe des Rechts erzwingen noch durch Narrative herbeierzählen lässt. Dazu benutzte er einen eingängigen, aber treffenden Vergleich: "[E]inen Baum kann man nicht bauen, man kann ihn nur pflanzen, pflegen und warten, dass er reift. " 107 Ebenso wenig kann mit einem harmonisierten Strafrecht ein einheitliches Rechtsgefühl oder eine gemeinsame Identität geschaffen oder genauer: erzwungen werden. ${ }^{108}$

\footnotetext{
103 Vgl. auch Vogel, in: Festschrift für Kühl, 2014, S. 635, 642.

$104 \mathrm{~V}_{\mathrm{g}}$. Europäische Kommission, Grünbuch über die Angleichung, die gegenseitige Anerkennung und die Vollstreckung strafrechtlicher Sankrionen in der Europäischen Union, KOM (2004) 334 endg., S. 10. Kritisch Eser, in: BMJ (Hrsg.), Rechtsraum Europa, 2002, S. 53, 72 f.; Frisch GA 2007, 250, 265; Vogel GA 2003, 314, 317; Weigend, in: Festschrift für Sootak, 2008, S. $243,251 \mathrm{f}$.

105 So Rat der Europäischen Union, Stockholm Programm: Für ein offenes und sicheres Europa im Dienste und zum Schutz der Bürger", Ratsdokument $17024 / 09$, S. 28.

106 Vogel GA 2003, 314, 319. Kritisch Satzger, in: Böse (Fn. 34), \ 2 Rn. 34 .

107 T.S. Eliot, Die Einheit der europäischen Kultur, 1946, S. 39.

108 Dazu bereits Kubiciel ZIS 2010, 742, 748.
}

\section{Ausblick}

Dem europäischen Gesetzgeber ist gleichermaßen Mut und Behutsamkeit anzuraten. Mutig zu schützen hat er europäische Institutionen, welche den Bürgern die Ausübung von Freiheit in der Union ermöglichen. Dazu bedarf es einheitlicher Normen, die gleichmäßig und wirksam, das heißt idealiter von einer Europäischen Staatsanwaltschaft durchgesetzt werden müssen. ${ }^{109}$ Aber auch Institutionen der Mitgliedstaaten, namentlich deren Legislative und Exekutive, sind vor Schädigungen von innen und außen zu schützen, weil die politischen, wirtschaftlichen und sozialen Folgen von Institutionenversagen auch andere Mitgliedstaaten erfassen. Eine kohärente und konsequente Korruptionsbekämpfung in allen Sektoren von Staat und Gesellschaft ist folglich eine genuin europäische Aufgabe. ${ }^{110}$ Gleiches gilt für den Schutz der Stabilität der Finanzwirtschaft. Darüber hinaus können bereits vereinheitlichte Verbraucherschutzregeln für die auf dem europäischen Markt tätigen Unternehmen strafrechtlich flankiert werden. Ob sich aus all dem am Ende ein weitgehend harmonisiertes europäisches Strafrecht entwickeln wird, vermag heute niemand zu sagen. Es ist zwar unbestreitbar, dass die Strafrechtsetzung in den letzten Jahrzehnten europäisiert und supranationalisiert worden ist.111 Daraus lässt sich jedoch nicht ableiten, dass diese Entwicklung eine ungebrochene Fortsetzung finden wird - und soll. Denn die von Liszt immer wieder vorgetragene These, wegen der „kausalen Gesetzmäßigkeit allen Geschehens, auch auf dem Gebiet des gesellschaftlichen Zusammenlebens ", ließe sich vom gewordenen und seienden Recht auf das künftig seinsollende Recht schließen, ${ }^{112}$ ist eine Rechnung mit zwei Unbekannten. Unklar ist schon, ob der schleichende schrittweise Abschied vom Staat als maßgeblichen Rechtssetzer unumkehrbar ist - viele Signale deuten gegenwärtig in eine andere Richtung. Vor allem aber lässt sich aus der bisherigen Europäisierung des Strafrechts - einem Sein - nicht auf ein künftiges Sollen schließen. Daher bedarf die Erzählung von einer fortschreitenden Europäisierung und Internationalisierung des Strafrechts ${ }^{113}$ einer Ergänzung: Es ist zu zeigen, dass das Strafrecht der EU eine gemeinsame Strafrechtsidee fortschreibt, deren Bewahrer die Staaten Europas (gewesen) sind und die behutsam auf die europäische Ebene zurückzuführen ist.

109 Zu Letzterem Esser StV 2014, 494 ff.; Schramm JZ 2014, 749 ff.

110 Zur Korruptionsbekämpfung in der EU Kubiciel HRRS 2013, $213 \mathrm{ff.}$ 111 Meyer (Fn. 87), S. $28 \mathrm{ff.}$

112 Siehe nur v. Liszt ZStW 26 (1906), 553, 556; ders. JW 1918, 754; ders. Vom Völkerbund zur Staatengemeinschaft, 1918, S. 5 f. Zum Garzen Herrmann (Fn. 5), S. 185 ff., 190: „Er (der Entwicklungsgedanke) stellt gleichsam das Leitmotiv in seinen wissenschaftlichen Bemühungen dar." 113 Vgl. zuletzt Meyer (Fn. 87), S. $604 \mathrm{ff}$. 